\section{Seltene Manifestationen von Nahrungsmittelallergien}

\author{
Neben den bekannten Manifestationsformen können Nahrungs- \\ mittelallergien auch seltsame und überraschende Symptome zur \\ Folge haben, die dem behandelnden Allergologen detektivisches \\ Gespür abverlangen.
}

A uf Grund eigener Erfahrung und nach Durchsicht der englischsprachigen Literatur bietet sich nachfolgende Dreiteilung ungewöhnlicher Manifestationen von Nahrungsmittelallergien an.

\section{Larvierte Allergien}

- Psyllium, ein Abführmittelbestandteil, kann als Verunreinigung in Mehlen vorkommen.

-Alpha-Amylase von Aspergillus oryzae oder Bacillus subtilis kommt in gebackenen Nahrungsmitteln vor, möglicherweise auch in Stärke, Getränken, Honig und diätetischen Nahrungsmitteln.

- Milch wurde in angeblich milchfreiem Früchtesorbet nachgewiesen.

-Allergische Reaktionen auf Anisakis simplex und andere Nematoden in Fischen werden besonders in Spanien beobachtet. Tieffrieren über 48 Stunden oder zehnminütiges Erhitzen über $60^{\circ} \mathrm{C}$ vernichtet die Allergenizität der Parasiten.

_Latex kann mit der Nahrung aufgenommen werden, wenn die zubereitenden Personen Latexhandschuhe getragen haben.

-Zahlreiche Nahrungsmitteladditiva - in den USA sind über 2.900 registriert - können Allergien oder Pseudo-Allergien hervorrufen.

\section{Ungewöhnliche Eintrittspforten - nichtallergische Einflussformen}

Erdnussstäube, die beim Knacken und Verarbeiten von Erdnüsse entstehen, können Asthma und Rhinitis hervorrufen. Ausdünstungen verschiedenster Nahrungsmittel, wie sie beispielsweise beim Kochen von Kartoffeln und Fisch entstehen, können Atemwegsallergien Nüssen kann eine Pro- hervorrufen. Berufliche Milch- und Eipulverinhalationen bei der Nahrungsmittelherstellung wurden wiederholt als Ursache einer Allergie identifiziert. Hautkontakt mit Fisch oder auch teindermatitis hervorrufen. Milcheiweiße in Salben haben eine ähnliche Wirkung auf die Haut. Carragen in Röntgenbrei wurde als intestinales Allergen entdeckt.

\section{Seltene Manifesta- tionsformen}

— Eine chronisch seröse Otitis media kann Folge einer Nahrungsmittelallergie sein. Wahrscheinliche Schockorgane sind die Eustachische Röhre und das

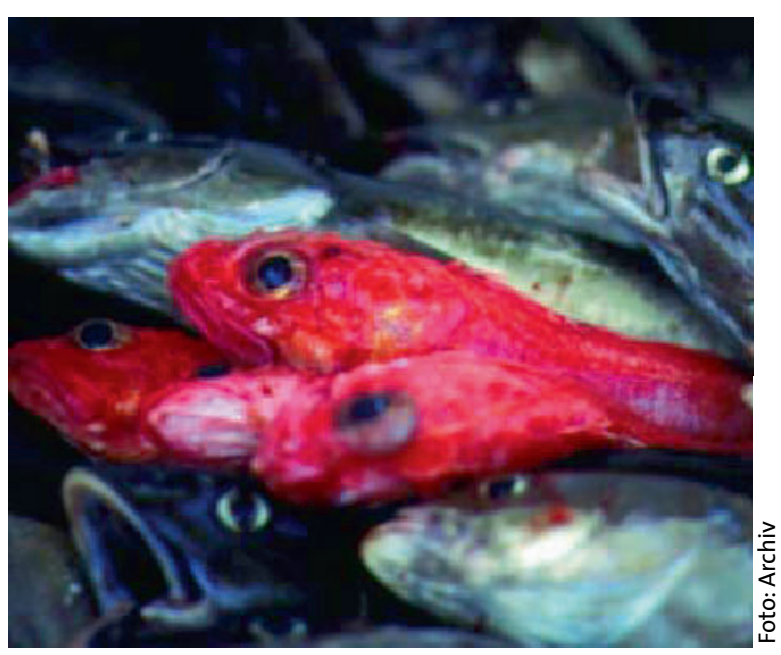

Diagnostische Herausforderung: In Fischen lebende Fadenwürmer können beim Verzehr ihres Wirtes zu allergischen Reaktionen führen.

Mittelohr selbst.

— In der Pädiatrie kann sich eine Milchallergie als Heiner-Syndrom der Kleinkinder sowie auch als Obstipation bei Kindern äußern. Auch kann Schlaflosigkeit bei Kindern eine allergische Ursache haben. Sie bessert sich unter hypoallergener Kost.

_ Die eosinophile Ösophagitis mit Reflux bei Milch- und Sojaallergie ist eine weitere seltene Organmanifestation.

— Besonders schwer zu diagnostizieren ist die Vaskulitis ausgelöst durch Additiva. Die Diagnose wird durch eine drei- bis vierwöchige hypoallergene Kost gestellt. — Das fixe juckende Erythem bei Linsenallergie ist ebenfalls nicht allgemein bekannt.

\section{Literatur}

1. Bahna SL. Unusual presentations of food allergy. Ann Allergy Asthma Immunol 2001; 86: $414-20$

Impressum „Der ÄDA informiert"

Verantwortlich für den Inhalt: Prof. Dr. Ludger Klimek, Wiesbaden Redaktion: Markus Seidl

Urban \& Vogel Medien und Medizin Verlagsgesellschaft $\mathrm{mbH} \& \mathrm{Co}$. KG Neumarkter Straße 43 81637 München

ÄDA-Geschäftsstelle: Service Systems Carin Fresle, Ursula Raab E-Mail:aeda@sersys.de
—Weitere vorkommende Raritäten sind Arthropathie und Synovitis, Proteinurie sowie Thrombozytopenie, jeweils bei Milchallergie.

\section{Schlussfolgerung}

Bei ätiologisch obskuren Symptomen sollte an eine Nahrungsmittelallergie gedacht werden. Anamnese, kontrollierte Verlaufsbeobachtung, Auslassversuche und Provokationsproben sind für dieses schwierige Gebiet der Allergologie besonders wichtig.

Dr. Dieter Bruchhausen, Wuppertal;

Prof. Dr. Joachim Sennekamp, Bonn 